\title{
ON A MULTIPLIER CONJECTURE FOR UNIVALENT FUNCTIONS
}

\author{
V. GRUENBERG, F. RØNNING, AND ST. RUSCHEWEYH
}

ABSTRACT. Let $\mathscr{S}$ be the set of normalized univalent functions, and let $\mathscr{D}$ be the subset of $\mathscr{S}$ containing functions with the property:

$$
\left|f^{\prime \prime}(z)\right| \leq \operatorname{Re} f^{\prime}(z), \quad|z|<1 .
$$

We present and discuss the following conjecture: For $f \in \mathscr{D}, g, h \in \overline{\operatorname{co}}(\mathscr{S})$,

$$
\operatorname{Re} \frac{1}{z}(f * g * h)(z)>0, \quad|z|<1 .
$$

In particular, we prove that the conjecture holds with $\mathscr{S}$ replaced by $\mathscr{C}$, the class of close-to-convex functions, and show its truth for a number of special members of $\mathscr{D}$. These latter results are extensions of old ones of Szegö and Kobori about sections of univalent functions.

\section{INTRODUCTION}

Let $\mathscr{A}_{0}$ be the set of analytic functions $f$ in the unit disk $\mathbb{D}$ normalized by $f(0)=0, f^{\prime}(0)=1$, and let $\mathscr{S}, \mathscr{C}, \mathscr{S}^{*}$ denote the subsets of $\mathscr{A}_{0}$ whose members are univalent, close-to-convex, and starlike in $\mathbb{D}$, respectively. Furthermore, let

$$
\mathscr{F}:=\overline{\mathrm{co}}\left\{\sum_{k=1}^{n} x^{k-1} z^{k}|| x \mid \leq 1, n \in \mathbb{N}\right\},
$$

and

$$
\mathscr{D}:=\left\{f \in \mathscr{A}_{0}|| f^{\prime \prime}(z) \mid \leq \operatorname{Re} f^{\prime}(z), \quad z \in \mathbb{D}\right\},
$$

where $\overline{\mathrm{co}}$ denotes the closed convex hull (in the topology of local uniform convergence in $\mathbb{D}$ ). In [6] the following conjecture was stated (* denotes the Hadamard product):

Conjecture A. Let $f \in \mathscr{D}, g \in \overline{\mathrm{co}}(\mathscr{S})$. Then $f * g \in \mathscr{S}^{*}$.

This conjecture is stronger than the former Bieberbach conjecture (de Branges' Theorem, [2]), since obviously

$$
f(z)=z+z^{n} / n^{2} \in \mathscr{D}
$$

Received by the editors December 5, 1988.

1980 Mathematics Subject Classification (1985 Revision). Primary 30C45, 30C55.

Research supported by the Fondo Nacional de Desarollo Científico y Tecnológico (FONDECYT, Grant 249/87), by the Universidad F. Santa Maria (Grant 88.12.06), by the German Academic Exchange Service (DAAD), and by the University of Trondheim AVH. 
and, for $g(z)=\sum_{k=1}^{\infty} a_{k} z^{k} \in \mathscr{S}$,

$$
(f * g)(z)=z+a_{n} z^{n} / n^{2} \text {. }
$$

The functions on the right-hand side of (1.2) are in $\mathscr{S}^{*}$ if and only if $\left|a_{n} / n^{2}\right| \leq$ $1 / n$, i.e., $\left|a_{n}\right| \leq n$.

In [6] the following result, supporting Conjecture A, was established.

Theorem A. For $F \in \mathscr{F}$ we have $f(z)=4 F(z / 4) \in \mathscr{D}$, and Conjecture A holds for $f$.

Theorem A represents a considerable improvement of the classical result of Szegö [8], which says that all partial sums of the power series expansion about $z=0$ of a function in $\mathscr{S}$ are univalent in $|z|<\frac{1}{4}$.

On the other hand, the occurrence of $\mathscr{S}^{*}$ in Conjecture A seems to be somewhat unmotivated (which one could say about the definition of $\mathscr{D}$ as well). To make things more transparent we point out the following equivalence:

Lemma 1.1. Let $H \in \mathscr{A}_{0}$. Then we have

$$
\left|\frac{H(z)}{z}-H^{\prime}(z)\right| \leq \operatorname{Re}\left(\frac{H(z)}{z}+H^{\prime}(z)\right), \quad z \in \mathbb{D},
$$

if and only if

$$
\operatorname{Re} \frac{1}{z}(H * h)(z)>0, \quad z \in \mathbb{D},
$$

holds for all $h \in \mathscr{C}$.

Proof. It suffices to study equation (1.4) for the extreme points

$$
h_{\alpha}(z)=\frac{1}{1+i \alpha}\left[\frac{z}{(1-z)^{2}}+i \alpha \frac{z}{1-z}\right], \quad \alpha \in \mathbb{R},
$$

of the closed convex hull of $\mathscr{C}$ (see [4, p. 56]). (1.4) is then seen to be equivalent to

$$
\operatorname{Re} \frac{H^{\prime}(z)+i \alpha H(z) / z}{1+i \alpha}>0, \quad \alpha \in \mathbb{R},
$$

which, in turn, is equivalent to (1.3).

The proof of Theorem A in [6] shows precisely that for $f$ as in the theorem, $g \in \mathscr{S}$, and $H=f * g$, we have (1.3). Hence, we have (equivalently)

$$
\operatorname{Re} \frac{1}{z}(f * g * h)(z)>0, \quad z \in \mathbb{D},
$$

for $f$ as in Theorem A, $g \in \mathscr{S}, h \in \mathscr{C}$. Clearly, by taking convex combinations, we deduce that (1.6) extends to $g \in \overline{\mathrm{co}}(\mathscr{S}), h \in \overline{\mathrm{co}}(\mathscr{C})$. Removing the somewhat unnatural unsymmetry in this statement, we are led to the following conjecture, which is considerably stronger than Conjecture A, but seems to be more natural.

Conjecture B. Let $f \in \mathscr{D}, g, h \in \overline{\operatorname{co}}(\mathscr{S})$. Then

$$
\operatorname{Re} \frac{1}{z}(f * g * h)(z)>0, \quad z \in \mathbb{D} \text {. }
$$


What remains is to motivate the occurrence of the multiplier family $\mathscr{D}$ in Conjectures A and B. Assume $f$ satisfies (1.7) for all $g, h \in \overline{\mathrm{co}}(\mathscr{S})$. Hence, choosing

$$
g(z)=\frac{z}{(1-z)^{2}}, \quad h \in \mathscr{C}, \quad H=f * g=z f^{\prime},
$$

we get from Lemma 1.1

$$
\left|z f^{\prime \prime}(z)\right| \leq \operatorname{Re}\left(z f^{\prime \prime}+2 f^{\prime}\right), \quad z \in \mathbb{D},
$$

as a necessary condition for $f$ (which is obviously satisfied for $f \in \mathscr{D}$ ).

Numerical calculations show that $f_{r}(z)=-\log (1-r z) / r$ satisfies $(1.9)$ for $r=0.656$ and $f_{r} \in \mathscr{D}$ for $r \leq \frac{1}{2}$. On the other hand, one can find functions $g$ and $h \in \mathscr{C}$ such that for $|z|=0.6174$

$$
\operatorname{Re} \frac{1}{z}\left(f_{r} * g * h\right)(z)<0 .
$$

This shows that in Conjecture B the condition for $\mathscr{D}$ cannot be replaced by (1.9), but also that the condition for $\mathscr{D}$ is not sharp for the conjecture. (This could not be expected anyway. It is sharp for some members of $\mathscr{D}$.)

The aim of the present paper is to support Conjecture B through a number of partial results, some of which are generalizations of known theorems in univalent function theory. First, we shall show (Theorem 2.1) that Conjecture B holds for $g, h \in \overline{\mathrm{co}}(\mathscr{C})$ (instead of $\overline{\mathrm{co}}(\mathscr{S})$ ). To this end, we also have to establish a general property of the functions in $\mathscr{D}$. All this will be done in $\S 2$.

Second, we shall generalize Theorem A to the extent of the new conjecture. This gives another refinement of Szegö's classic result $(\S 3)$.

In $\S 4$, we shall show that the functions

$$
f_{n}(z)=\sum_{k=1}^{n} \frac{z^{k}}{k 2^{k-1}}, \quad n \in \mathbb{N},
$$

are in $\mathscr{D}$. This, in connection with the truth of Conjecture B for close-to-convex functions, establishes a new result on the partial sums for those functions that extends a theorem of Kobori [5]. We are not able, so far, to prove Conjecture $\mathrm{B}$ for $f_{n}$ as in (1.10), in general. In the special case $g=z(1-z)^{-2}$ (the Koebe function), however, we can do so: if $h(z)=\sum_{k=1}^{\infty} a_{k} z^{k} \in \mathscr{S}$, then

$$
\operatorname{Re} \sum_{k=1}^{n} a_{k} z^{k-1}>0, \quad|z|<\frac{1}{2}, n \in \mathbb{N} .
$$

This is sharp in the sense that the number $\frac{1}{2}$ cannot be replaced by a larger one $(\S 5)$.

One general remark should be in order about the use of numerical work in our proofs. We are frequently led to the problem of showing that certain polynomials are positive in some interval. In some of these cases we used standard root-finding algorithms (IMSL library with MS-FORTRAN 4.01) to verify the nonvanishing of the polynomials in the interval of interest. We here 
adopt the point of view that carefully obtained numerical evidence in this kind of problem is as good a proof as evidence obtained from the application of classic calculus.

\section{THE CLOSE-TO-CONVEX CASE}

We first establish the following properties of the functions in $\mathscr{D}$.

Lemma 2.1. For $f \in \mathscr{D}$ and $z \in \mathbb{D}$ we have

$$
\left|f^{\prime}(z)-\frac{f(z)}{z}\right| \leq|z| \min \left\{\operatorname{Re} f^{\prime}(z), \operatorname{Re} \frac{f(z)}{z}\right\} .
$$

Proof. Since

$$
f^{\prime}(z)-\frac{f(z)}{z}=\int_{0}^{1} t z f^{\prime \prime}(t z) d t
$$

and $\left|f^{\prime \prime}(z)\right| \leq \operatorname{Re} f^{\prime}(z)$, it suffices to estimate

$$
a(z):=\operatorname{Re} \int_{0}^{1} t f^{\prime}(t z) d t .
$$

Integration by parts yields

$$
\begin{aligned}
a(z) & =\frac{1}{2} \operatorname{Re} f^{\prime}(z)-\operatorname{Re} \frac{z}{2} \int_{0}^{1} t^{2} f^{\prime \prime}(t z) d t \\
& \leq \frac{1}{2} \operatorname{Re} f^{\prime}(z)+\frac{1}{2} \int_{0}^{1} t\left|f^{\prime \prime}(t z)\right| d t \\
& \leq \frac{1}{2} \operatorname{Re} f^{\prime}(z)+\frac{1}{2} a(z),
\end{aligned}
$$

which gives $a(z) \leq \operatorname{Re} f^{\prime}(z)$. On the other hand,

$$
a(z) \leq \operatorname{Re} \int_{0}^{1} f^{\prime}(t z) d t=\operatorname{Re} \frac{f(z)}{z}
$$

and (2.1) is established.

We mention in passing that a more careful analysis yields the following estimate for $f \in \mathscr{D}$ :

$$
\left|f^{\prime}(z)-\frac{f(z)}{z}\right| \leq \frac{1}{|z|}\left(e^{|z|}-1-|z|\right) \operatorname{Re} f^{\prime}(z) \leq(e-2) \operatorname{Re} f^{\prime}(z), \quad z \in \mathbb{D} .
$$

Theorem 2.1. For $f \in \mathscr{D}, g, h \in \overline{\mathrm{co}}(\mathscr{C})$ we have

$$
\operatorname{Re} \frac{1}{z}(f * g * h)(z)>0, \quad z \in \mathbb{D} \text {. }
$$

Proof. It clearly suffices to prove Theorem 2.1 for the extreme points of $\overline{c o}(\mathscr{C})$, more explicitly, for (see (1.5))

$$
g=h_{\alpha}, \quad h=h_{\beta}, \quad \alpha, \beta \in \mathbb{R} .
$$

Note that the set of all extreme points of $\overline{c o}(\mathscr{C})$ consists of the functions $h_{\alpha}(x z) / x$ where $|x|=1$ and $\alpha \in \mathbb{R} \backslash\{0\}$. The rotations in the argument, however, do not affect (2.2). 
Evaluating the convolution in (2.2) leads us in this case to the equivalent condition:

$$
\operatorname{Re} \frac{A+i(\alpha+\beta) B}{1+i(\alpha+\beta)} \frac{-\alpha \beta C}{-\alpha \beta}>0
$$

where

$$
A=A(z)=z f^{\prime \prime}(z)+f^{\prime}(z), \quad B=B(z)=f^{\prime}(z), \quad C=C(z)=\frac{f(z)}{z} .
$$

We note that, in view of Lemma 2.1 and the definition of $\mathscr{D}$, we may write

$$
\begin{array}{lll}
A=B+\varepsilon \operatorname{Re} B, & & |\varepsilon| \leq 1, \\
C=B+\delta \operatorname{Re} B, & & |\delta| \leq 1 .
\end{array}
$$

Thus

$$
\operatorname{Re} \frac{A+i(\alpha+\beta) B-\alpha \beta C}{1+i(\alpha+\beta)-\alpha \beta}=\operatorname{Re} B\left[1+\operatorname{Re} \frac{\varepsilon-\alpha \beta \delta}{1+i(\alpha+\beta)-\alpha \beta}\right],
$$

and it suffices to show that

$$
\left|\frac{\varepsilon-\alpha \beta \delta}{1+i(\alpha+\beta)-\alpha \beta}\right| \leq 1
$$

for $\alpha, \beta \in \mathbb{R}, \varepsilon, \delta \in \overline{\mathbb{D}}$, which is a simple exercise in calculus.

Remark. $\overline{\mathrm{co}}(\mathscr{C})$ contains all normalized typically real functions. Hence, Theorem 2.1 implies the truth of Conjecture B for all $g, h \in \mathscr{S}$ with real Taylor coefficients.

\section{EXTENSION OF SZEGÖ'S THEOREM}

It is not difficult to see that

$$
f_{n}(z)=4 \sum_{k=1}^{n}\left(\frac{z}{4}\right)^{k} \in \mathscr{D}, \quad n \in \mathbb{N},
$$

(compare Theorem A), and we wish to verify Conjecture B for the functions $f=f_{n}$, which is easily seen to be equivalent to

Theorem 3.1. Let $g(z)=\sum_{k=1}^{\infty} a_{k} z^{k} \in \mathscr{S}, h(z)=\sum_{k=1}^{\infty} b_{k} z^{k} \in \mathscr{S}$. Then, for $n \in \mathbb{N}$, we have

$$
\operatorname{Re} \sum_{k=1}^{n} \frac{a_{k} b_{k}}{4^{k-1}}>0
$$

The number 4 in (3.1) cannot be replaced by any smaller positive number.

Note that the choices $h(z)=z(1-x z)^{-2}, x \in \mathbb{D}$, lead to the conclusion that

$$
\operatorname{Re}\left(\sum_{k=1}^{n} a_{k}\left(\frac{x}{4}\right)^{k}\right)^{\prime}>0, \quad x \in \mathbb{D}
$$


which implies that the partial sums of $g$ are univalent in $|z|<\frac{1}{4}$, Szegö's result. Of course, (3.1) is much stronger than that.

The proof of Szegö's theorem (compare Duren [3, p. 243]) splits into the more or less elementary cases $n=2, n \geq 4$, and the case $n=3$, for which the much deeper Loewner theory was required. In our proof of Theorem 3.1 it is also the case $n=3$ that is the hard one. However, we show that the elementary area theorem has enough information, thereby giving a means to simplify even Szegö's original proof.

In the proof we have to use a number of results about $\mathscr{S}$ that we now state as a lemma.

Lemma 3.1. Let $g(z)=\sum_{k=1}^{\infty} a_{k} z^{k} \in \mathscr{S}$. Then we have

$$
\begin{aligned}
\left|\log \frac{g(z)}{z}+\log \left(1-|z|^{2}\right)\right| & \leq \log \frac{1+|z|}{1-|z|}, \quad z \in \mathbb{D}, \\
\left|a_{k}\right| & \leq k, \quad k \in \mathbb{N}, \\
\left|a_{3}-\frac{3}{4} a_{2}^{2}\right| & \leq \frac{2}{\sqrt{3}} \sqrt{1-\left|\frac{a_{2}}{2}\right|^{2}} .
\end{aligned}
$$

Equation (3.2) is due to Grunsky (cf. [3, p. 323]); for (3.3) see de Branges [2]. Equation (3.4) is a consequence of the area theorem (see [3, p. 132]).

We shall also want the following result.

Lemma 3.2. For $j=1,2$ let $H_{j}$ be analytic in $\mathbb{D}, H_{j}(0)=1$ and $\operatorname{Re} H_{j}(z)>$ $\alpha, z \in \mathbb{D}$. Then

$$
\operatorname{Re}\left(H_{1} * H_{2}\right)(z)>4 \alpha-2 \alpha^{2}-1, \quad z \in \mathbb{D} .
$$

Proof. Let

$$
\widetilde{H}_{1}:=\frac{1}{2} \frac{H_{1}-\alpha}{1-\alpha}+\frac{1}{2} .
$$

Then $\widetilde{H}_{1}(0)=1, \operatorname{Re} \widetilde{H}_{1}(z)>\frac{1}{2}, z \in \mathbb{D}$. It is well known that these functions preserve the convex hull of the image domain of a given function under convolution, i.e., $\operatorname{Re}\left(\widetilde{H}_{1} * H_{2}\right)(z)>\alpha, z \in \mathbb{D}$. The result follows immediately.

We now turn to the proof of Theorem 3.1. We have to distinguish the cases $n=2,3$, and $n \geq 4$. However, the case $n=2$ is trivial, but also shows that the number 4 in the theorem cannot be replaced by a smaller one.

The case $n \geq 4$. From (3.2) we deduce that

$$
\operatorname{Re} \frac{g(z)}{z} \geq \min _{|\rho| \leq 1} \frac{4}{3} \operatorname{Re}\left(3^{\rho}\right)>0.43714, \quad|z| \leq \frac{1}{2},
$$

as a numerical evaluation of the minimum shows.

The same inequality holds for $h$, and Lemma 3.2 applied to

$$
H_{1}(z)=\frac{g(z / 2)}{z / 2}, \quad H_{2}(z)=\frac{h(z / 2)}{z / 2}
$$


and $\alpha=0.43714$ yields

$$
\operatorname{Re}\left(H_{1} * H_{2}\right)(1)=\operatorname{Re} \sum_{k=1}^{\infty} \frac{a_{k} b_{k}}{4^{k-1}} \geq 0.36637 .
$$

We note that this bound is surely not the best one; however, it cannot be improved to more than 0.38400 since this is the value of

$$
\sum_{k=1}^{\infty} k^{2}\left(-\frac{1}{4}\right)^{k-1}
$$

Now we are using the following estimate:

$$
\begin{aligned}
\operatorname{Re} \sum_{k=1}^{n} \frac{a_{k} b_{k}}{4^{k-1}} & =\operatorname{Re} \sum_{k=1}^{\infty} \frac{a_{k} b_{k}}{4^{k-1}}-\operatorname{Re} \sum_{k=n+1}^{\infty} \frac{a_{k} b_{k}}{4^{k-1}} \\
& \geq 0.36637-\sum_{k=n+1}^{\infty} \frac{k^{2}}{4^{k-1}},
\end{aligned}
$$

and a simple evaluation shows that this is positive for $n \geq 4$.

The case $n=3$. This case depends heavily on the following technical lemma.

Lemma 3.3. Let $0 \leq \alpha \leq \frac{2}{3}, \quad x_{0}(\alpha):=\frac{1}{2}\left(1-\alpha / \sqrt{1+\alpha^{2}}\right)$, and $\lambda(x)=x+$ $\alpha \sqrt{1-x^{2}}$. Then, for $x_{0}(\alpha) \leq A \leq x \leq 1$, we have

$$
\lambda(x) \lambda(A / x) \leq \lambda^{2}(\sqrt{A}) .
$$

Remark. The lower bound for $A$ is sharp. It is likely that the result holds for all $\alpha \geq 0$, but the technical problems in the proof increase with $\alpha$. We restrict ourselves therefore to the cases that we really need.

Proof. Let $\mu(x)=\lambda(x) \lambda(A / x)$. Then

$$
\frac{x \mu^{\prime}(x)}{\mu(x)}=\frac{\alpha}{\alpha+\nu(A / x)}-\frac{\alpha}{\alpha+\nu(x)}
$$

with

$$
\nu(x)=x \sqrt{1-x^{2}}-\alpha x^{2} .
$$

Hence, possible extrema for $\mu$ in the given interval are the points $x=1, \sqrt{A}$, $A$ and any $x$ with $x^{2} \neq A$ but satisfying $\nu(x)=\nu(A / x)$. The following proposition rules out the existence of such an $x$.

Proposition 3.1. Let $x_{1}, x_{2} \in[0,1], x_{1}<x_{2}$, and $\nu\left(x_{1}\right)=\nu\left(x_{2}\right)$. Then $x_{1} \cdot x_{2}<x_{0}(\alpha)$.

Proof of the proposition. $\nu^{\prime}$ has its only zero in the interval $[0,1]$ at $\tilde{x}=$ $\sqrt{x_{0}(\alpha)}$, and this corresponds to a maximum of $\nu$. Hence, we must have $x_{1}<\tilde{x}<x_{2}$. But we shall prove that

$$
\nu\left(\tilde{x}+\left(\tilde{x}-x_{1}\right)\right)<\nu\left(\tilde{x}-\left(\tilde{x}-x_{1}\right)\right)=\nu\left(x_{1}\right),
$$


which implies $\tilde{x}<x_{2}<2 \tilde{x}-x_{1}$, and thus

$$
x_{1} x_{2} \leq 2 \widetilde{x} x_{1}-x_{1}^{2}=\widetilde{x}^{2}-\left(\widetilde{x}-x_{1}\right)^{2}<\widetilde{x}^{2},
$$

the assertion. To prove (3.7), we write $\zeta=\tilde{x}-x_{1}$ and, using the fact that $\nu^{\prime}(\tilde{x})=0$, we see that

$$
\nu(\tilde{x}+\zeta)-\nu(\tilde{x}-\zeta)=\int_{0}^{\zeta} \int_{0}^{t} \int_{-s}^{s} \nu^{\prime \prime \prime}(\tilde{x}+\tau) d \tau d s d t<0
$$

since

$$
\nu^{\prime \prime \prime}(x)=-3\left(1-x^{2}\right)^{-5 / 2}<0 .
$$

To complete the proof of Lemma 3.3 it remains to show that

$$
\mu(1)=\mu(A) \leq \mu(\sqrt{A}), \quad x_{0}(\alpha) \leq A \leq 1 .
$$

This is equivalent to

$$
\rho(A):=\sqrt{1+\frac{1}{A}}-\alpha \sqrt{\frac{1}{A}-1} \leq 2 .
$$

Now we have

$$
\rho^{\prime \prime}(A)=\frac{(4 A+3)(1-A)^{3 / 2}+\alpha(4 A-3)(1+A)^{3 / 2}}{4 A^{5 / 2}\left(1-A^{2}\right)^{3 / 2}},
$$

and it is easy to see that for $0 \leq \alpha \leq \frac{2}{3}$, we have $\rho^{\prime \prime}(A)>0, A \in[0,1)$, which implies the convexity of $\rho(A)$ in that interval. Hence, equation (3.8) has to be checked only in the points $A=1$ (where it is trivial) and $A=x_{0}(\alpha)$. For $\alpha=0$ we have $\rho\left(x_{0}(\alpha)\right)=\sqrt{3}<2$. Furthermore,

$$
\frac{d}{d \alpha} \rho\left(x_{0}(\alpha)\right)=\frac{1}{\beta(\beta-\alpha)}\left[\sqrt{\frac{\beta-\alpha}{3 \beta-\alpha}}-\alpha-\beta\right], \quad \beta=\sqrt{\alpha^{2}+1},
$$

and a simple verification shows that this is negative for $\alpha \geq 0$. Hence, equation (3.8) holds, and the proof is complete.

We now write

$$
\gamma_{3}=\operatorname{Re}\left(1+\frac{a_{2} b_{2}}{4}+\frac{a_{3} b_{3}}{16}\right) .
$$

Using equation (3.4) we get

$$
\begin{array}{ll}
a_{3}=\frac{3}{4} a_{2}^{2}+\rho_{1} \frac{2}{\sqrt{3}} \sqrt{1-\left|\frac{a_{2}}{2}\right|^{2}}, & \left|\rho_{1}\right| \leq 1, \\
b_{3}=\frac{3}{4} b_{2}^{2}+\rho_{2} \frac{2}{\sqrt{3}} \sqrt{1-\left|\frac{b_{2}}{2}\right|^{2}}, & \left|\rho_{2}\right| \leq 1,
\end{array}
$$


and therefore

$$
\begin{aligned}
\gamma_{3} \geq & \operatorname{Re}\left(1+\frac{a_{2} b_{2}}{4}+\frac{9}{256}\left(a_{2} b_{2}\right)^{2}\right) \\
& -\frac{\sqrt{3}}{8}\left(\left|\frac{a_{2}}{2}\right|^{2} \sqrt{1-\left|\frac{b_{2}}{2}\right|^{2}}+\left|\frac{b_{2}}{2}\right|^{2} \sqrt{1-\left|\frac{a_{2}}{2}\right|^{2}}\right) \\
& -\frac{1}{12} \sqrt{1-\left|\frac{a_{2}}{2}\right|^{2}} \sqrt{1-\left|\frac{b_{2}}{2}\right|^{2}} .
\end{aligned}
$$

Now we write

$$
\left|a_{2}\right|=2 x, \quad\left|b_{2}\right|=2 y, \quad a_{2} b_{2}=4 A e^{i \varphi}, \quad A=x y .
$$

Then, applying $|x| \leq 1,|y| \leq 1$, we get

$$
\begin{aligned}
\gamma_{3} \geq 1 & +A \cos \varphi+\frac{9}{16} A^{2} \cos (2 \varphi)-\frac{\sqrt{3}}{8}\left(x \sqrt{1-y^{2}}+y \sqrt{1-x^{2}}\right) \\
& -\frac{1}{12} \sqrt{1-x^{2}} \sqrt{1-y^{2}} \\
= & +A \cos \varphi+\frac{9}{16} A^{2} \cos (2 \varphi) \\
& -\frac{9}{16}\left(x+\frac{2}{3 \sqrt{3}} \sqrt{1-x^{2}}\right)\left(y+\frac{2}{3 \sqrt{3}} \sqrt{1-y^{2}}\right)+\frac{9}{16} A .
\end{aligned}
$$

We now minimize with respect to $\varphi$ and apply Lemma 3.3 with $\alpha=\frac{2}{3 \sqrt{3}}=$ $0.38 \ldots$ This yields

$$
\gamma_{3} \geq \frac{7}{9}-\frac{9}{16} A^{2}- \begin{cases}\frac{9}{16}\left[\left(\sqrt{A}+\frac{2}{3 \sqrt{3}} \sqrt{1-A}\right)^{2}-A\right], & A \geq \frac{1}{2}-\frac{1}{\sqrt{31}} \\ \frac{\sqrt{3}}{4}+\frac{1}{12}, & A<\frac{1}{2}-\frac{1}{\sqrt{31}}\end{cases}
$$

In the case below we get $\gamma_{3}>0.2>0$. Evaluation in the upper case leaves us with

$$
\gamma_{3} \geq \frac{25}{36}+\frac{1}{12} A-\frac{9}{16} A^{2}-\frac{1}{4} \sqrt{3 A(1-A)},
$$

and we have to show that this is nonnegative for $\frac{1}{2}-\frac{1}{\sqrt{31}} \leq A \leq 1$. Taking squares we see that this condition is satisfied if

$$
P(A):=6561 A^{4}-1944 A^{3}-12168 A^{2}-1488 A+10000>0
$$

for $0 \leq A \leq 1$. But $P(0)>0$, and $P$ has its root at $-0.968348 \pm 0.513761 i$ and $1.116496 \pm 0.147723 i$ (all given digits precise), and we are done.

\section{INTEGRATED PARTIAL SUMS}

The main goal of this section is to identify more members in $\mathscr{D}$. This leads to some new results on close-to-convex functions (via Theorem 2.1) and to new candidates for checking Conjecture B.

Theorem 4.1. For $n \in \mathbb{N}$ we have

$$
f_{n}(z):=\sum_{k=1}^{n} \frac{z^{k}}{k 2^{k-1}} \in \mathscr{D} .
$$


The number 2 cannot be replaced by any smaller positive number without violating the result.

Remark. It is interesting that in this case the sharpness of the result does not only come from the elementary case $n=2$, but also from $n \rightarrow \infty$. This fact makes the proof harder.

Proof. As before, the proof is to be split into the cases of a few small $n$ and the remainder, in this case, $n \geq 7$. This time, however, the latter case is the more difficult one. The cases $n \leq 6$ can be left to numerical verification, after some suitable analytic preparation.

We must show that $\left|f_{n}^{\prime \prime}(z)\right| \leq \operatorname{Re} f_{n}^{\prime}(z), z \in \mathbb{D}$. We note that, since $\left|f_{n}^{\prime \prime}\right|-$ $\operatorname{Re} f_{n}^{\prime}$ is a subharmonic function, we can restrict our attention to the boundary. The inequality is then easily seen to be equivalent to

$$
\frac{1}{2}\left|1-n z^{n-1}+(n-1) z^{n}\right| \leq \operatorname{Re}\left(1-z+\frac{1}{4} z^{n-1}-z^{n}\right), \quad|z|=\frac{1}{2} .
$$

Writing

$$
z=\frac{1}{2} e^{i \varphi}, \quad x=\cos \varphi, \quad T_{n}(x)=\cos (n \varphi),
$$

where $T_{n}$ are the Chebyshev polynomials, we see that (4.1) is equivalent to $\rho(x) \leq \sigma(x)$, where

$$
\begin{aligned}
& \rho(x):=\frac{1}{2^{n+1}}\left(2^{2 n}+4 n^{2}+(n-1)^{2}-\right. 4 n(n-1) x \\
&\left.+2^{n+1}\left((n-1) T_{n}(x)-2 n T_{n-1}(x)\right)\right)^{\frac{1}{2}}, \\
& \sigma(x):=1-\frac{1}{2} x+\frac{1}{2^{n+1}} T_{n-1}(x)-\frac{1}{2^{n}} T_{n}(x),
\end{aligned}
$$

and $-1 \leq x \leq 1$. Since $\sigma(x)>0$, we can square both sides, and we are eventually led to the polynomial inequality $P_{n}(x) \geq 0,-1 \leq x \leq 1$, where

$$
\begin{aligned}
P_{n}(x):= & 3-4 x+x^{2}+\frac{4 n(n-1)}{2^{2 n}} x \\
& +\frac{1}{2^{n-1}}\left((2-x+2 n) T_{n-1}(x)-(3-2 x+n) T_{n}(x)\right) \\
& +\frac{1}{2^{2 n}}\left(T_{n-1}(x)-2 T_{n}(x)\right)^{2}-\frac{4 n^{2}+(n-1)^{2}}{2^{2 n}} .
\end{aligned}
$$

It is clear from the way $P_{n}(x)$ was obtained (there was a denominator $|1-z|^{2}$ removed to get $(4.1))$ that each $P_{n}$ has a factor $(4 x-5)^{2}$. Writing $P_{n}(x)=$ $2^{-2 n-2}(4 x-5)^{2} H_{n}(x)$ we obtain

$$
H_{n}(x)=\sum_{k=0}^{2 n-2} c_{k}^{(n)} x^{k}
$$


TABLE 1

\begin{tabular}{|c|c|c|c|c|c|c|c|c|c|c|c|}
\hline$n$ & $c_{0}^{(n)}$ & $c_{1}^{(n)}$ & $c_{2}^{(n)}$ & $c_{3}^{(n)}$ & $c_{4}^{(n)}$ & $c_{5}^{(n)}$ & $c_{6}^{(n)}$ & $c_{7}^{(n)}$ & $c_{8}^{(n)}$ & $c_{9}^{(n)}$ & $c_{10}^{(n)}$ \\
\hline 2 & 3 & 4 & 1 & & & & & & & & \\
\hline 3 & 1 & 4 & 16 & 8 & 4 & & & & & & \\
\hline 4 & 19 & -44 & 1 & 56 & 24 & 32 & 16 & & & & \\
\hline 5 & 149 & -28 & -316 & -48 & 240 & 32 & 64 & 128 & 64 & & \\
\hline 6 & 531 & 548 & -159 & -1872 & -520 & 1120 & 304 & -128 & 128 & 512 & 256 \\
\hline
\end{tabular}

The coefficients $c_{k}^{(n)}$, for $n=2,3,4,5,6$, can be read from Table 1 .

Table 2 shows the roots of $H_{n}(x), 2 \leq n \leq 6$, with all given digits precise.

TABLE 2. Roots of the polynomials $H_{n}(x)$

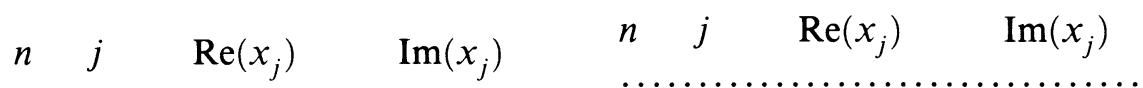

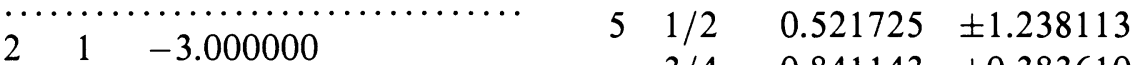

$$
\begin{aligned}
& \begin{array}{lllll}
2 & -1.000000 & 3 / 4 & -0.841143 & \pm 0.383610
\end{array} \\
& \begin{array}{lll}
5 / 6 & 0.722206 & \pm 0.179647
\end{array} \\
& 7 / 8 \quad-1.402788 \quad \pm 0.869921 \\
& 3 \quad 1 / 2 \quad-0.125315 \quad \pm 0.236497 \\
& 3 / 4-0.874684 \pm 1.650710
\end{aligned}
$$

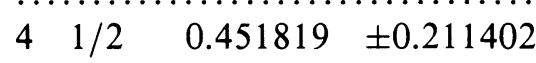

$$
\begin{aligned}
& 3-1.153210 \\
& \begin{array}{ll}
4 & -1.911702
\end{array} \\
& \begin{array}{lll}
5 / 6 & 0.080637 & \pm 1.469082
\end{array} \\
& \begin{array}{rrrr}
6 & 1 & -1.164235 & \\
2 / 3 & -0.394230 & \pm 0.505396 \\
4 / 5 & 0.764047 & \pm 1.056512 \\
6 / 7 & -0.808437 & \pm 1.154491 \\
8 / 9 & 0.861445 & \pm 0.147145 \\
10 & -1.681414 &
\end{array}
\end{aligned}
$$

These numbers supply sufficient evidence to support the statement that $H_{n}(x)$ $\neq 0,-1 \leq x \leq 1$, and, since $H_{n}(0)>0$, we have $H_{n}(x)>0$ in that interval, $n=2, \ldots, 6$.

We now turn to the proof of $P_{n}(x)>0,-1 \leq x \leq 1$, for $n \geq 7$.

Proposition 4.1. $P_{n}(x)>0$ holds for $n \geq 7, \cos \frac{\pi}{n} \leq x \leq 1$.

Proof. In this interval we have $T_{n-1}(x) \geq T_{n}(x)$ and hence:

$$
(2-x+2 n) T_{n-1}(x)-(3-2 x+n) T_{n}(x) \geq(-1+x+n) T_{n}(x) .
$$

Thus,

$$
P_{n}(x) \geq 3-4 x+x^{2}+\frac{4 n(n-1)}{2^{2 n}} x-\frac{4 n^{2}+(n-1)^{2}}{2^{2 n}}+\frac{n-1+x}{2^{n-1}} T_{n}(x)
$$

We begin with the subinterval $\cos \frac{\pi}{2 n+2} \leq x \leq 1$. We omit the positive term $3-4 x+x^{2}$ and observe that the rest of the right-hand side of (4.2) is increasing 
with $x$. Hence, we have to study only $x=\cos \frac{\pi}{2 n+2}$, which leaves us with the following inequality:

$$
\cos \frac{n \pi}{2 n+2}+\frac{4 n}{2^{n+1}} \cos \frac{\pi}{2 n+2}-\frac{4 n^{2}+(n-1)^{2}}{(n-1) 2^{n+1}}>0 .
$$

It is easy to show that (4.3) holds for $n \geq 7$, and we omit the details.

Next we turn to the subinterval $\cos \frac{\pi}{2 n} \leq x \leq \cos \frac{\pi}{2 n+2}$. This time we delete from the right-hand side the nonnegative $T_{n}(x)$, and we see that it suffices to prove

$$
3-4 x+x^{2} \geq \frac{4 n^{2}+(n-1)^{2}}{2^{2 n}} .
$$

Writing $x=\cos y$ and using $\cos y \leq 1-\frac{1}{2} y^{2}+\frac{1}{24} y^{4},|y| \leq 1$, we obtain

$$
3-4 x+x^{2}=(3-\cos y)(1-\cos y) \geq y^{2},
$$

and it remains to show that

$$
\frac{\pi^{2}}{4(n+1)^{2}} \geq \frac{4 n^{2}+(n-1)^{2}}{2^{2 n}}
$$

which is correct for $n \geq 7$.

Finally, let $\cos \frac{\pi}{n} \leq x \leq \cos \frac{\pi}{2 n}$. Now $T_{n}(x) \leq 0$. Using (4.4) and writing $z=n y$ (such that $\left.\frac{\pi}{2} \leq z \leq \pi\right)$, we see that $P_{n}(x)>0$ is implied by

$$
z^{2}+2 \frac{n^{3}}{2^{n}} \cos z \geq 4\left(\frac{n^{2}}{2^{n}}\right)^{2}+\frac{1}{2} \frac{(n-1)^{2}}{2^{n-1}} \frac{n^{2}}{2^{n}} .
$$

We note that $n^{3} / 2 n$ and $n^{2} / 2^{n}$ are decreasing with $n$ for $n \geq 5$. Since $\cos z \leq 0$, the worst case in (4.5) occurs for the smallest value of $n$ in question, i.e., $n=7$ :

$$
z^{2}+\frac{343}{64} \cos z \geq \frac{11368}{16384}, \quad \frac{\pi}{2} \leq z \leq \pi .
$$

Since this is true, the proof of Proposition 4.1 is complete.

Proposition 4.2. $P_{n}(x) \geq 0$ holds for $n \geq 7, \cos \frac{3 \pi}{2 n} \leq x \leq \cos \frac{\pi}{n}$.

Proof. In equation (4.5) we have seen that

$$
Q_{n}(x):=3-4 x+x^{2}+\frac{n}{2^{n-1}} T_{n}(x)-\frac{4 n^{2}+(n-1)^{2}}{2^{2 n}} \geq 0
$$

is true for $x=\cos \frac{\pi}{n}$. But $Q_{n}^{\prime}(x)<0$ in the present interval, and therefore $Q_{n}(x) \geq 0, \cos \frac{3 \pi}{2 n} \leq x \leq \cos \frac{\pi}{n}$. An inspection of the definition of $P_{n}(x)$ shows that $P_{n}(x) \geq Q_{n}(x)$ as long as $T_{n-1}(x) \geq T_{n}(x)$ and $T_{n}(x) \leq 0$, which is fulfilled for a certain interval $\cos \frac{3 \pi}{2 n} \leq x_{n} \leq x \leq \cos \frac{\pi}{n}$, with $T_{n-1}\left(x_{n}\right)=$ $T_{n}\left(x_{n}\right)$. Hence, $P_{n}(x) \geq 0$ for $x_{n} \leq x \leq \cos \frac{\pi}{n}$. In the remaining interval, $\cos \frac{3 \pi}{2 n} \leq x \leq x_{n}$, we have $T_{n-1}(x) \leq T_{n}(x) \leq 0$, and therefore

$$
P_{n}(x) \geq 3-4 x+x^{2}+\frac{n}{2^{n-1}} T_{n-1}(x)-\frac{4 n^{2}+(n-1)^{2}}{2^{2 n}}
$$


in this interval. The right-hand side is obviously larger than $Q_{n}\left(\cos \frac{\pi}{n}\right)$, which is nonnegative.

The rest of the proof of Theorem 4.1 is easy. Replacing $T_{n}, T_{n-1}$ in the definition of $P_{n}$ by the worst possibilities in $[-1,1]$ we are left with

$$
P_{n}(x) \geq 3-4 x+x^{2}-\frac{1}{2^{n-1}}(5-3 x+3 n)-\frac{4 n^{2}+(n-1)^{2}}{2^{2 n}},
$$

which we have to show to be nonnegative for $-1 \leq x \leq \cos \frac{3 \pi}{2 n}$. The worst case occurs at the right extremum. Using (4.4) and $\cos y \geq 1-\frac{1}{2} y^{2}$ we reduce the problem to

$$
\left(\frac{3 \pi}{2 n}\right)^{2}\left(1-\frac{3}{2 n}\right)-\frac{2+3 n}{2^{n-1}}-\frac{4 n^{2}+(n-1)^{2}}{2^{2 n}}>0 .
$$

This latter inequality holds for $n \geq 7$.

A combination of Theorems 2.1 and 4.1 yields

Theorem 4.2. Let

$$
g(z)=\sum_{k=1}^{\infty} a_{k} z^{k} \in \overline{\mathrm{co}}(\mathscr{C}), \quad h(z)=\sum_{k=1}^{\infty} b_{k} z^{k} \in \overline{\mathrm{co}}(\mathscr{C}) .
$$

Then, for $n \in \mathbb{N}$,

$$
\operatorname{Re} \sum_{k=1}^{n} \frac{a_{k} b_{k}}{k} z^{k-1}>0, \quad|z|<\frac{1}{2} .
$$

Corollary 4.1. Let $g(z)=\sum_{k=1}^{\infty} a_{k} z^{k} \in \overline{\mathrm{co}}(\mathscr{C})$. Then

$$
2 \sum_{k=1}^{n} \frac{a_{k}}{k}\left(\frac{z}{2}\right)^{k} \in \mathscr{S}^{*}
$$

This result extends considerably a theorem of Kobori [5], who proved the same result only for $g \in \mathscr{S}^{*}$. Note that $\overline{\mathrm{co}}(\mathscr{C})$ contains, for instance, also all typically real functions. Note also that the same conclusion holds for arbitrary convex combinations of functions of the form (4.6) (with $n$ varying).

\section{CONJeCtURE B FOR INTEgRated PARTIAL SUMS}

We now prove the following theorem:

Theorem 5.1. Let $h(z)=\sum_{k=1}^{\infty} a_{k} z^{k} \in \mathscr{S}$. Then, for $n \in \mathbb{N}$,

$$
\operatorname{Re} \sum_{k=1}^{n} a_{k} z^{k-1}>0, \quad|z|<\frac{1}{2} .
$$

From this we immediately get the following corollary. 
Corollary 5.1. Let $h(z)=\sum_{k=1}^{\infty} a_{k} z^{k} \in \mathscr{S}$. Then

$$
2 \sum_{k=1}^{n} \frac{a_{k}}{k}\left(\frac{z}{2}\right)^{k} \in \mathscr{S} \text {. }
$$

We note that this result is another extension of the previously mentioned theorem of Kobori. Besides, it is a verification of Conjecture B in the special case that $f_{n}$ is as in $(1.10)$ and $g(z)=z(1-z)^{-2}$. We are not able to prove Conjecture B for arbitrary $g \in \mathscr{S}$, and in fact we do not even know whether

$$
\operatorname{Re} \sum_{k=1}^{\infty} \frac{a_{k} b_{k}}{k}\left(\frac{1}{2}\right)^{k-1}>0
$$

for $a_{k}, b_{k}$ coefficients of functions in $\mathscr{S}$.

Proof. The proof will be split into several cases according to the value of $n$. We note that the case $n=2$ is trivial, and also that the result is sharp for this (and no other) value of $n$.

The case $n \geq 6$. Because the class $\mathscr{S}$ is preserved under rotations and (certain) dilations, it is enough to prove (5.1) in the point $z=\frac{1}{2}$. In (3.5) we used the result of Grunsky, (3.2), to calculate the minimum of $\operatorname{Re} g(z) / z$ for $|z| \leq \frac{1}{2}$. Using this again we get

$$
\begin{aligned}
\operatorname{Re} \sum_{k=1}^{n} a_{k}\left(\frac{1}{2}\right)^{k-1} & \geq 0.43714-\operatorname{Re} \sum_{k=n+1}^{\infty} a_{k}\left(\frac{1}{2}\right)^{k-1} \\
& \geq 0.43714-\sum_{k=n+1}^{\infty} k\left(\frac{1}{2}\right)^{k-1} .
\end{aligned}
$$

Computing the right-hand side of (5.2), we find that it is positive for $n \geq 6$.

The case $n=3$. We are now going to prove

$$
\operatorname{Re}\left(2 a_{2}+a_{3}\right) \geq-4 \text {. }
$$

Using, (3.4), we get

$$
\operatorname{Re}\left(2 a_{2}+a_{3}\right) \geq \operatorname{Re}\left(2 a_{2}+\frac{3}{4} a_{2}^{2}\right)-\frac{2}{\sqrt{3}} \sqrt{1-\left|\frac{a_{2}}{2}\right|^{2}} .
$$

Substituting $a_{2}=2 r e^{i \varphi}$, we see that (5.3) will follow from

$$
6 r^{2} \cos ^{2} \varphi+4 r \cos \varphi-3 r^{2}+4 \geq \frac{2}{\sqrt{3}} \sqrt{1-r^{2}}
$$

Calculating the minimum of the left-hand side with respect to $\varphi$, we find that this is attained for

$$
\cos \varphi= \begin{cases}-1 & \text { if } r<\frac{1}{3} \\ -\frac{1}{3 r} & \text { if } r \geq \frac{1}{3}\end{cases}
$$


Hence, we are left with the inequalities

$$
\begin{array}{rlrl}
3 r^{2}-4 r+4 & \geq \frac{2}{\sqrt{3}} \sqrt{1-r^{2}}, & r<\frac{1}{3}, \\
\frac{10}{3}-3 r^{2} \geq \frac{2}{\sqrt{3}} \sqrt{1-r^{2}}, & r \geq \frac{1}{3} .
\end{array}
$$

We easily check that these are both fulfilled, and hence (5.3) is established.

The case $n=4$. We now proceed to prove the inequality

$$
\operatorname{Re}\left(2 a_{2}+a_{3}+\frac{a_{4}}{2}\right) \geq-4,
$$

which gives Theorem 5.1 for $n=4$. We now get an estimate on $\operatorname{Re} a_{4}$ using the following inequality, which can be found in the book of Bieberbach [1, p. 118]. (We assume $\left|a_{2}\right|<2$.)

$$
\begin{gathered}
\left|\sqrt{3} \sqrt{1-\left|\frac{a_{2}}{2}\right|^{2}}\left(-\frac{a_{4}}{2}+a_{2} a_{3}-\frac{13}{24} a_{2}^{3}\right)-\sqrt{3} \frac{\bar{a}_{2}}{2} \frac{\left(\frac{a_{3}}{2}-\frac{3}{8} a_{2}^{2}\right)^{2}}{\sqrt{1-\left|\frac{a_{2}}{2}\right|^{2}}}\right| \\
\leq \frac{1-\left|\frac{a_{2}}{2}\right|^{2}-3\left|\frac{a_{3}}{2}-\frac{3}{8} a_{2}^{2}\right|^{2}}{\sqrt{3} \sqrt{1-\left|\frac{a_{2}}{2}\right|^{2}}} .
\end{gathered}
$$

Equation (5.5) is derived from the Grunsky inequalities, and it is used in [1] for the proof of $\left|a_{4}\right| \leq 4$. From (5.5) we get

$$
\begin{aligned}
\operatorname{Re} \frac{a_{4}}{2} & \geq \operatorname{Re}\left(a_{2} a_{3}-\frac{13}{24} a_{2}^{3}\right)-\frac{1}{3}+\frac{\left|\frac{a_{3}}{2}-\frac{3}{8} a_{2}^{2}\right|^{2}}{1-\left|\frac{a_{2}}{2}\right|^{2}}-\operatorname{Re} \frac{\bar{a}_{2}}{2} \frac{\left(\frac{a_{3}}{2}-\frac{3}{8} a_{2}^{2}\right)^{2}}{1-\left|\frac{a_{2}}{2}\right|^{2}} \\
& \geq-\frac{1}{3}+\operatorname{Re}\left(a_{2} a_{3}-\frac{13}{24} a_{2}^{3}\right)+\left(1-\left|\frac{a_{2}}{2}\right|\right) \frac{\left|\frac{a_{3}}{2}-\frac{3}{8} a_{2}^{2}\right|^{2}}{1-\left|\frac{a_{2}}{2}\right|^{2}} \\
& \geq-\frac{1}{3}+\operatorname{Re}\left(a_{2} a_{3}-\frac{13}{24} a_{2}^{3}\right) .
\end{aligned}
$$

Now (5.4) will follow if we can prove

$$
\operatorname{Re}\left(2 a_{2}+a_{3}+a_{2} a_{3}-\frac{13}{24} a_{2}^{3}\right) \geq-\frac{11}{3} \text {. }
$$

Applying (3.4), we get an expression not involving $a_{3}$.

$$
\begin{gathered}
\operatorname{Re}\left(2 a_{2}+a_{3}+a_{2} a_{3}-\frac{13}{24} a_{2}^{3}\right) \geq \operatorname{Re}\left(2 a_{2}+\frac{3}{4} a_{2}^{2}+\frac{5}{24} a_{3}^{2}\right)-\left(1+\left|a_{2}\right|\right)\left|a_{3}-\frac{3}{4} a_{2}^{2}\right| \\
\geq \operatorname{Re}\left(2 a_{2}+\frac{3}{4} a_{2}^{2}+\frac{5}{24} a_{2}^{3}\right)-\frac{2}{\sqrt{3}}\left(1+\left|a_{2}\right|\right) \sqrt{1-\left|\frac{a_{2}}{2}\right|^{2}} .
\end{gathered}
$$

Finally, we prove

$$
\operatorname{Re}\left(2 a_{2}+\frac{3}{4} a_{2}^{2}+\frac{5}{24} a_{2}^{3}\right)+\frac{11}{3} \geq \frac{2}{\sqrt{3}}\left(1+\left|a_{2}\right|\right) \sqrt{1-\left|\frac{a_{2}}{2}\right|^{2}},
$$


where $a_{2}$ is an arbitrary complex number with $\left|a_{2}\right|<2$. Now (5.7) will imply (5.6), which again will imply (5.4).

Substituting $a_{2}=2 r e^{i \varphi},(5.7)$ turns into

$$
\frac{5}{3} r^{3} \cos (3 \varphi)+3 r^{2} \cos (2 \varphi)+4 r \cos \varphi+\frac{11}{3} \geq \frac{2}{\sqrt{3}}(1+2 r) \sqrt{1-r^{2}} .
$$

The right-hand side here is always positive, so by squaring both sides we get the equivalent relation (where we have put $x:=r \cos \varphi$ and $y:=r^{2}$ ):

$$
\begin{aligned}
\left(225 x^{2}\right. & +270 x+129) y^{2}-\left(600 x^{4}+900 x^{3}+684 x^{2}+498 x+234\right) y \\
& +400 x^{6}+720 x^{5}+804 x^{4}+872 x^{3}+540 x^{2}+216 x+109 \geq 0 .
\end{aligned}
$$

The left-hand side of (5.8) is a parabola $c_{2} y^{2}+c_{1} y+c_{0}, c_{2}>0$. This takes its minimum value for $y=-c_{1} / 2 c_{2}$, and if this minimum value is positive for all $x \in(-1,1)$, then we are done. Substituting $y=-c_{1} / 2 c_{2}$ into (5.8) we get

$$
\frac{4\left(1600 x^{6}+4080 x^{5}+4116 x^{4}+2606 x^{3}+873 x^{2}-81 x+31\right)}{75 x^{2}+90 x+43} \geq 0 \text {. }
$$

The denominator is always positive, so we have to show

$$
\begin{aligned}
P(x):= & 1600 x^{6}+4080 x^{5}+4116 x^{4}+2606 x^{3} \\
& +873 x^{2}-81 x+31 \geq 0, \quad x \in(-1,1) .
\end{aligned}
$$

The roots of $P$ are as follows: $-1.060003,-1.120350,-0.253773 \pm 0.747233 i$, and $0.068950 \pm 0.146435 i$. Now $P(0)>0$, and from the list of roots we see that $P(x)$ has no zeros in the interval $(-1,1)$, hence $P(x)>0$ for all $x \in(-1,1)$. This completes the proof in the case $n=4$.

The case $n=5$. The proof for this case is again split in two. First, consider (5.2), which for $n=5$ takes the form

$$
\operatorname{Re} \sum_{k=1}^{5} a_{k}\left(\frac{1}{2}\right)^{k-1} \geq 0.18714-\frac{1}{32} \operatorname{Re} a_{6}
$$

The right-hand side of this inequality is positive if $\operatorname{Re} a_{6}<5.988$. Hence, we have established Theorem 4.3 for $n=5$ and $h \in \mathscr{S}$ with $\operatorname{Re} a_{6}<5.988$. Using a similar argument we can prove the theorem if $\operatorname{Re} a_{7}<6.976$. To complete the case $n=5$, we now assume that we have a function $h(z)=\sum_{k=1}^{\infty} a_{k} z^{k}$ with $\operatorname{Re} a_{6} \geq 5.988$ and $\operatorname{Re} a_{7} \geq 6.976$. We use the following lemma:

Lemma 5.1. Let $f(z)=\sum_{k=1}^{\infty} a_{k} z^{k} \in \mathscr{S}$. Then

$$
\operatorname{Re} a_{5} \geq 5 \operatorname{Re}\left(a_{6}+a_{7}\right)-63 \text {. }
$$

Now we see that if $\operatorname{Re} a_{6} \geq 5.988$ and $\operatorname{Re} a_{7} \geq 6.976$, it follows from the lemma that $\operatorname{Re} a_{5} \geq 1.82$. In particular $\operatorname{Re} a_{5}>0$, and hence (5.1) for $n=5$ follows from (5.4). 
This lemma is an application of a result stated by Sheil-Small [7] as a conjecture. He shows that this conjecture is contained in the Robertson conjecture on odd univalent functions. Since this now is known to be true by de Branges' Theorem, we may use Sheil-Small's conjecture as a result.

Lemma 5.2. Let $p$ be a polynomial of degree $\leq n$ and let $f \in \mathscr{S}$. Then

$$
\|f * p\| \leq n\|p\| \text {. }
$$

(II $\|$ denotes the maximum modulus norm in $\mathbb{D}$.)

Proof of Lemma 5.1. Let $p(z)=z^{5}-5 z^{6}-5 z^{7}$. An elementary calculation shows that $\|p\|=9$. With $h(z)=\sum_{k=1}^{\infty} a_{k} z^{k}$ we get $(h * p)(z)=a_{5} z^{5}-$ $5 a_{6} z^{6}-5 a_{7} z^{7}$, and by Lemma 5.2,

$$
\left|a_{5}-5 a_{6}-5 a_{7}\right| \leq\|f * p\| \leq 7\|p\|=63,
$$

and hence

$$
\operatorname{Re} a_{5} \geq 5 \operatorname{Re}\left(a_{6}+a_{7}\right)-63 .
$$

With the proof of Lemma 5.1, the proof of Theorem 5.1 is complete.

\section{REFERENCES}

1. L. Bieberbach, Einführung in die konforme Abbildung, De Gruyter, Berlin, 1967.

2. L. de Branges, A Proof of the Bieberbach Conjecture, Acta Math. 154 (1985), 137-152.

3. P. L. Duren, Univalent functions, Springer-Verlag, 1983.

4. D. J. Hallenbeck and T. H. MacGregor, Linear problems and convexity techniques in geometric function theory, Pitman, 1984.

5. A. Kobori, Zwei Sätze über die Abschnitte schlichter Potenzreihen, Mem. Coll. Kyoto 17 (1934), 172-186.

6. St. Ruscheweyh, Extension of Szegö's theorem on the sections of univalent functions, SIAM J. Math. Anal. 19 (1988), 1442-1449.

7. T. Sheil-Small, On the convolution of analytic functions, J. Reine Angew. Math. 258 (1973), 137-152.

8. G. Szegö, Zur Theorie der schlichten Abbildungen, Math. Ann. 100 (1928), 188-211.

Departamento de Matemática, Universidad Técnica f. Santa Marfa, Valparafso, CHILE

INSTITUTT FOR MATEMATIKK OG STATISTIKK, UNiVERSitetet I TRONDHEIM AVH, N-7055 DragVOLL, NoRWAY

Mathematisches Institut der Universität, D-8700 Würzburg, Federal Republic of GERMANY 